\title{
Effect of degree and location of intra-pair similarity upon paired-associate acquisition
}

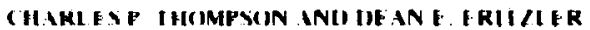

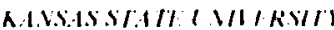

A systematic increase in rate of acquisition of a pairedassociate list was demonstrated as the degree to which formal similarity between stimulus and response items was located within, rather than between, pairs was increased. In addition, the results showed that identity of the first letters of the stimulus and response produced more rapid acquisition than identity of the last letters of the stimulus and response.

Although a great deal of effort has been spent in investigating the effects of similarity among the stimulus or response members in paired-associate (PA) learning, relatively little research has been concerned with similarity between stimulus and response members. Nevertheless, there is considerable evidence to suggest that $S-R$ similarity is a potent variable which may markedly facilitate or inhibit PA acquisition depending upon the particular arrangement of items in the list. For example, a double function PA list (one in which each item serves as both a stimulus and a response) is much more difficult to learn than a single function PA list containing the same number of pairs (Umemoto \& Hilgard, 1961). This result depends, of course, upon the restriction that the double function occur between, rather than within, palrs since the occurrence of the double function within pairs would make the list trivially easy. It seems likely that acquisition of a pair in a PA list also should be facilitated when the members of the pair are similar, although this facilitation will be less marked than when members of the pair are identical. Likewise, interference should occur when the locus of the S-R similarity is between pairs. Therefore, given some degree of similarity between the stimulus and response members of a PA list, the list should be learned more rapidly as the degree to which the similarity is located within, rather than between, pairs is increased.

In addition, the location in the stimulus terms of an element which is repeated in a response term could be quite important. For illustration, consider a trigram-trigram PA list with the stimulus trigrams picked to insure stimulus selection. Since the evidence (e.g., Postman \& Greenbloom, 1967) rather overwhelmingly suggests that, under these conditions, the first letter of the stimulus trigram will be selected as the functional stimulus, it seems reasonable to assume that repetition of this letter would produce the greatest facilitatory (within pair letter repetition) or inhibitory (between pair letter repetition) effects.
Repetition of the third stimulus letter, on the other hand, should have relatively little effect.

The present experiment tested the above hypotheses by manipulating the degree to which S-R similarity occurred within, rather than between, pairs and also by manipulating the location of the intrapair repeated elements.

\section{Subjects and Design}

A total of 133 undergraduate students, divided equally among the seven experimental conditions, served as Ss in the experiment. The seven conditions were obtained by varying the number of trigram pairs in a given list of 10 pairs which contained a repeated letter, and varying the location in the stimulus and response trigram of the repeated letter. Specifically, the seven conditions consisted of three levels of number of pairs containing repeated letters $(10,8$, and 4$)$ for each of two locations (first letter (F) of $S$ and $R$ terms identical; last letters (L) identical) plus a no repeated letter (NRL) condition.

Lists

One basic set of $10 \mathrm{CVC}$ trigram pairs was used to generate all the lists. All letters of the alphabet were used in generating the stimuli with each of the vowels (including $Y$ ) used once and none twice. The responses were generated by using all letters in the same location as in the stimulus terms (i.e., first, second, or third letter) with the restriction that no two letters appearing in any one $\mathrm{S}$ term appear in any one $\mathrm{R}$ term. The mean association value (Archer, 1960) was 25.8 for the stimulus trigrams and 42.6 for the response trigrams.

All lists were generated by appropriate pairings of this single set of stimulus and response trigrams. As a precaution against unique pairing effects, four different sublists were generated for each of the $8 \mathrm{~F}$, $8 \mathrm{~L}, 4 \mathrm{~F}, 4 \mathrm{~L}$, and NRL conditions.

\section{Procedure}

Group data collection procedures were utilized with small groups of four or five Ss run simultaneously. Ss were given standard instructions for study-test PA acquisition. The lists were presented on $3 \times 5$ cards at a 4 sec rate, with an intertrial interval of 12 sec. Twelve different orders of presentation were used to minimize serial learning. The lists were presented for 30 trials and $\mathrm{Ss}$ wrote the responses for each trial on a separate page in a small data booklet.

The data were tabulated in terms of trials and errors to a criterion of one errorless trial. In those 
Table 1

\begin{tabular}{lrrrrrrr}
\hline Condition & \multicolumn{1}{c}{$10 \mathrm{~F}$} & $10 \mathrm{~L}$ & $8 \mathrm{~F}$ & $8 \mathrm{~L}$ & $4 \mathrm{~F}$ & $4 \mathrm{~L}$ & NRL \\
\hline Mean errors & 31.8 & 49.0 & 49.6 & 62.3 & 56.3 & 68.1 & 81.9 \\
Mean trials & 7.4 & 11.0 & 12.1 & 14.4 & 14.4 & 15.2 & 16.5 \\
\hline
\end{tabular}

instances in which $S$ did not achieve criterion, total errors were tabulated and $S$ was assigned the arbitrary score of 31 trials to criterion.

\section{Results and Discussion}

Analyses performed on the total trials and total errors data showed the differences among the groups to be highly significant (trials $-F=3.68, \mathrm{df}=1 / 126$, $p<.01$; errors $-F=3.70, \mathrm{df}=1 / 126, p<.01$ ). The means for the trials and errors data are presented in Table 1. Additional analyses, excluding the NRL condition data, demonstrated that ease of PA acquisition increased as the number of pairs with a repeated letter within the pair increased (trials $-F=6.72$, df $=2 / 108, p<.01$; errors $-F=4.25$, $d f=2 / 108, p<.025)$. In addition, Ss made significantly fewer errors when the first letter was repeated than when the last letter was repeated $(F=5.29, \mathrm{df}=1 / 108, \mathrm{p}<.025)$.

We had assumed that those pairs containing a repeated letter (RL pairs) would be learned more rapidly than those which did not contain a repeated letter (NRL pairs). To evaluate this assumption more adequately, we tabulated the mean number of errors per RL and NRL pair for each $S$ in the four conditions (8F, 8L, 4F, and 4L) which contained both types of pairs. As expected, significantly fewer errors were made on RL pairs (5.04 errors/pair) than on NRL pairs (6.85 errors/pair; $F=7.99$, $d f=1 / 144, p<.01$ ). Again, Ss made fewer errors per pair when the first letter was repeated (5.31 errors/pair) than when the last letter was repeated (6.58 errors/pair; $F=3.96$, $\mathrm{df}=1 / 144, p<.05)$. No other source of variation was significant in the analysis of the errors/pair data.

Our data demonstrate that the location of $S-R$ similarity (between or within pairs) is a powerful variable which can have marked effects on the ease of PA acquisition. In addition, our results strongly suggest that the effects of S-R similarity are modified by the location within the trigrams of the repeated elements. Whether the relative facilitation produced by intrapair similarity results from the addition of a repeated letter or from reduced interpair similarity (or from both), and whether the effects due to location of the repeated letter may be attributed to stimulus location or to response location (or to both), is a matter for future investigation.

\section{References}

ARCHER, E. J. A re-evaluation of the meaningfulness of all possible CVC trigrams. Psychol. Monogr., 1960, 74, No. 10 (Whole No. 497). POSTMAN, L., \& GREENBLOOM, R. Conditions of cue selection in the acquisition of paired-associate lists. J. exp. Psychol, 1967, 73, 91-100.

UMEMOTO, T., \& HILGARD, E. R. Paired-associate learning as a function of similarity: Common stimulus and response items within the list. J. exp. Psychol., 1961, 62, 97-104.

Note

1. This research was supported in part by Public Health Service Research Grant MH 11598 from the National Institutes of Mental Health. 